\section{Birds in Kurigram district of Bangladesh}

\section{Shawkat Imam Khan ${ }^{1}$ \& Habibon Naher ${ }^{2}$}

\author{
${ }^{1}$ Department of Natural History, Bangladesh National Museum, \\ Dhaka 1000, Bangladesh \\ ${ }^{2}$ Lecturer, Department of Biology, Shahid Zia Girl's School and \\ College, Jatrabari, Dhaka 1204, Bangladesh \\ Email: ${ }^{1}$ sukhi_194@yahoo.com
}

This study is an attempt to document the diversity, status, habitat, distribution and some other aspects of birds in Kurigram District in Bangladesh. Past major works of avifauna of Bangladesh include those of Khan (2005), Thompson \& Johnson (2003), Islam (1970). The Kurigram District includes many islands or "Chars" making it difficult to assume the exact number of species of birds inhabiting these islands. These islands are of various sizes and they remain submerged during the monsoon and become exposed in winter. The area is very rich in faunal diversity, especially avifauna.

\section{Study area}

Kurigram $\left(89^{\circ} 28^{\prime}-89^{\circ} 50^{\prime} \mathrm{E} \& 25^{\circ} 20^{\prime}-26^{\circ} 15^{\prime} \mathrm{N}\right)$ is one of the northern districts of Bangladesh. The states of West Bengal and Assam in India border the north and east of Kurigram, respectively. Native district Gaibanda is to the south and Lalmonirhat and Rangpur districts are to the west. Kurigram District proper is $301 \mathrm{~km}$ by road and $307 \mathrm{~km}$ by rail from Dhaka. Total area of the district is $2296 \mathrm{~km}^{2}$ covering nine thanas (precincts). In the northern section of Kurigram is the flood plain of the Tista River while in the south is the GangaBrahmaputra basin. The north is at an altitude of $30 \mathrm{~m}$ while the rest is $10 \mathrm{~m}$. The Tista, the Brahmaputra and the Dharala are the major rivers crisscrossing the district (Fig. 1). The annual rainfall is $2200 \mathrm{~mm}$. The temperature ranges between $9.3^{\circ}$ and $36.7^{\circ} \mathrm{C}$.

\section{Materials and Methods}

Field surveys were conducted along the rivers Dharala and Brahmaputra in Kurigram District from November 2000 to February 2002 to document avifaunal diversity. Birds were recorded during the first and last weeks in each month, between

Date of publication 26 April 2009

ISSN 0974-7907 (online) | 0974-7893 (print)

Editor: C. Srinivasulu

\section{Manuscript details:}

Ms \# 01698

Received 30 December 2006

Final revised received 29 November 2007

Finally accepted 19 June 2008

Citation: Khan, S.I. \& H. Naher (2009). Birds in Kurigram district of Bangladesh. Journal of Threatened Taxa 1(4): 245-250.

Copyright: @ Shawkat Imam Khan \& Habibon Naher 2009. Creative Commons Attribution 3.0 Unported License. JoTT allows unrestricted use of this article in any medium for non-profit purposes, reproduction and distribution by providing adequate credit to the authors and the source of publication.

OPEN ACGESS | FREE DOWNLOAD

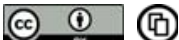

0630-1800hr everyday.

Existing roads, bridle paths, embankments, cattle trails, agricultural fields, riversides, rivers, canals and pond banks were used to cover the study area. All types of habitats were visited monthly. Most field visits were carried out on foot but sometimes vehicles were also used to cover long distances. A motorized boat was also used to cover the rivers and the canals.

Observations were made either with a pair of binoculars or by naked eyes depending on the distance of the bird. Standard literature (Ali 1996; Ali \& Ripley 1983; Grimmett et al. 1999) were followed for species identification, gait, flight pattern, habitat, call and sitting posture.

Status of the recorded birds was assessed on the basis of standard methods outlined by Khan (1980). The status categories are: Very Common (VC) - bird species which were recorded on $80-100 \%$ of the field visits; Common (C) - species that were observed on 50-79\% of the field visits; Fairly Common (FC) - species that were found on $20-49 \%$ of the field visits; and Rare $(\mathrm{R})$ - species that were found on less than $19 \%$ of the visits.

We determined the status of each bird species using calculations, in terms of percentage of occurrence, while earlier studies (Khan 1980, 1982) determined these categories based on assumption and experience. Resident and migratory species were recognized by consulting old records (IUCN 2000; Harvey 1990; Khan 1982) and through our observations.

Resident - species that are found year-round and some of which were seen to make nests;

Migratory - species recorded only at certain times of the year.

Three canopies (levels) of vegetation could be recognized in the study area:

1. Lower canopy: maximum height of about $4 \mathrm{~m}$ from the ground, including bushes, shrubs, small trees, and lower parts of big trees.

2. Middle canopy: 4-10m above the ground and

3. Upper canopy: above 10m high.

The bird species have been listed according to the taxonomic classification of Inskipp et al. (1996).

\section{Results and Discussion}

The avifaunal diversity of Bangladesh includes 632 species of birds belonging to 16 orders and 67 families (IUCN 2000). A total of $105(16.6 \%)$ species (Table 1 ) of birds were recorded in the Kurigram District, belonging to 12 orders, 35 families, and 77 genera. Recorded species comprised of $51(48.6 \%)$ non-passerine and $54(51.4 \%)$ passerine birds. Out of 105 species, $33(31.4 \%)$ were migratory and $72(68.6 \%)$ were resident. Among the 51 species of non-passerine birds, 15 $(29.4 \%)$ migratory and $36(70.6 \%)$ were resident, while, among the 54 species of passerine birds, 18 (33.3\%) were migratory and $36(66.7 \%)$ were resident.

During the study period, out of 105 species, $14(13.3 \%)$ were found to be very common, 30 (28.6\%) common, $25(23.8 \%)$ fairly common and $36(34.3 \%)$ rare or few.

During observations, it was noticed that many species used more than one habitat. Out of 105 species, 30 (28.6\%) species used aquatic and semi-aquatic habitats including river, 


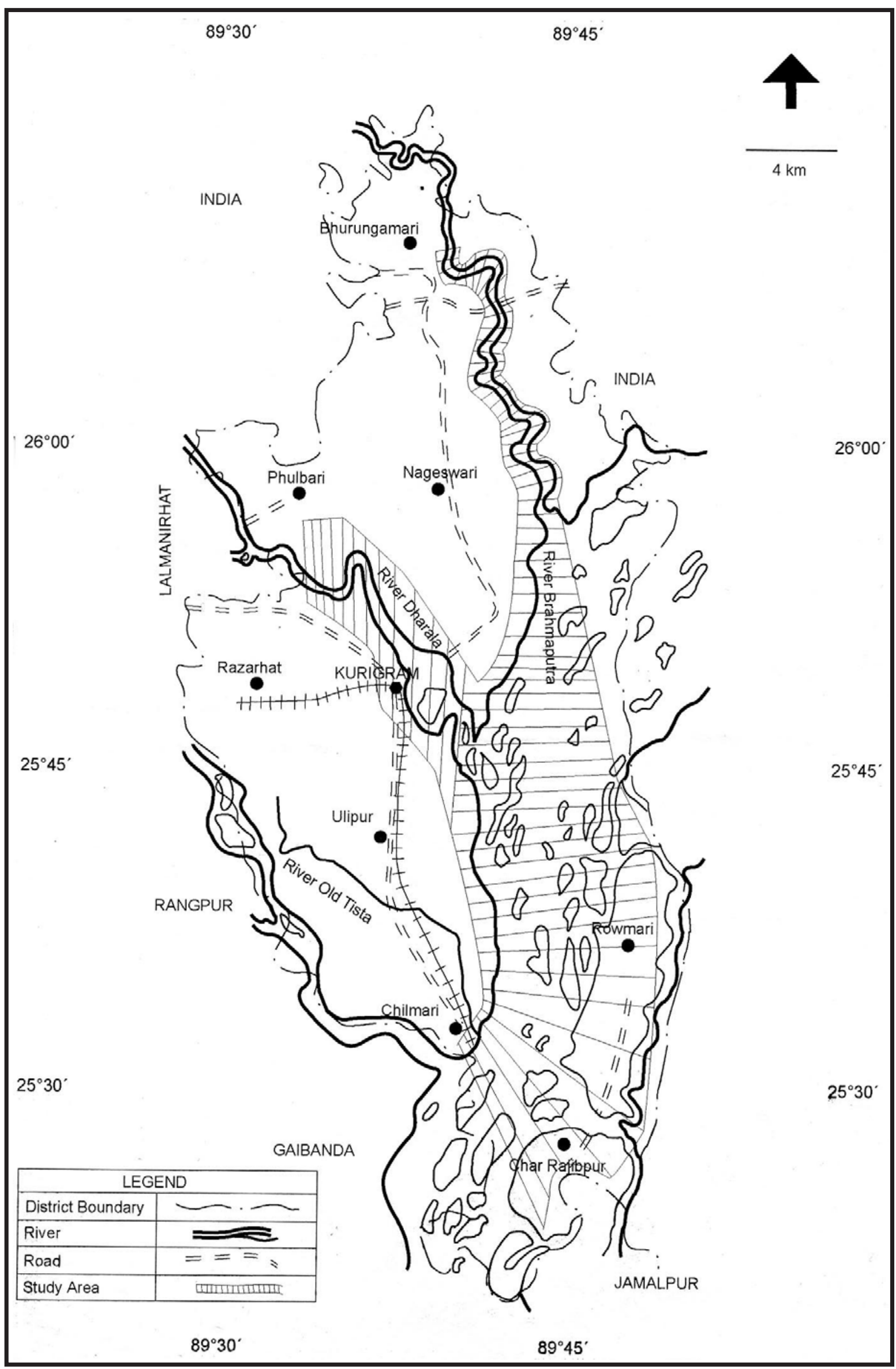

Figure 1. Map of the study area 
Table 1. Systematic list of the bird species recorded along the Dharala and Brahmaputra rivers in Kurigram District, Bangladesh from November 2000 to February 2002.

\begin{tabular}{|c|c|c|c|c|c|}
\hline Scientific name & Common Name & $\begin{array}{l}\text { Migratory (M) / } \\
\text { Resident (R) }\end{array}$ & Habitat & Local status & Local Distribution \\
\hline \multicolumn{6}{|l|}{$\begin{array}{l}\text { Order: Anseriformes } \\
\text { Family: Anatidae }\end{array}$} \\
\hline Anser anser & Greylag Goose & M & $r$, re & $\mathrm{Ra}$ & NE \\
\hline Tadorna ferruginea & Ruddy Shelduck & M & r, re & $\mathrm{Fc}$ & NE \\
\hline Anas platyrhynchos & Mallard & M & $r$ & $\mathrm{Ra}$ & NE \\
\hline \multicolumn{6}{|l|}{$\begin{array}{l}\text { Order: Piciformes } \\
\text { Family: Picidae }\end{array}$} \\
\hline Dinopium benghalense & Black-rumped Flameback & $\mathrm{R}$ & $\mathrm{T}$ & Vc & W \\
\hline Celeus brachyurus & Rufous woodpecker & $\mathrm{R}$ & $J$ & $\mathrm{Fc}$ & Cn \\
\hline \multicolumn{6}{|l|}{ Family: Megalaimidae } \\
\hline Megalaima asiatica & Blue-throated Barbet & $\mathrm{R}$ & $\mathrm{T}$ & $\mathrm{C}$ & Cn, NW \\
\hline Megalaima rubricapilla & Crimson-fronted Barbet & $\mathrm{R}$ & $\mathrm{T}$ & $\mathrm{C}$ & W \\
\hline Megalaima haemacephala & Coppersmith Barbet & $\mathrm{R}$ & $\mathrm{T}$ & $\mathrm{Fc}$ & NW \\
\hline \multicolumn{6}{|l|}{$\begin{array}{l}\text { Order: Upupiformes } \\
\text { Family: Upupidae }\end{array}$} \\
\hline Upupa epops & Common Hoopoe & $\mathrm{R}$ & V & $\mathrm{Ra}$ & NE \\
\hline \multicolumn{6}{|l|}{$\begin{array}{l}\text { Order: Coraciiformes } \\
\text { Family: Alcedinidae }\end{array}$} \\
\hline Alcedo atthis & Common Kingfisher & $\mathrm{R}$ & $\mathrm{T}, \mathrm{We}$ & $\mathrm{C}$ & W \\
\hline Alcedo meninting & Blue-eared Kingfisher & $\mathrm{R}$ & $\mathrm{J}, \mathrm{We}$ & $\mathrm{Ra}$ & $\mathrm{Cn}$ \\
\hline Halcyon smyrnensis & White-throated Kingfisher & $\mathrm{R}$ & $\mathrm{V}, \mathrm{J}, \mathrm{r}$ & $\mathrm{C}$ & W \\
\hline $\begin{array}{l}\text { Family: Cerylidae } \\
\text { Ceryle rudis }\end{array}$ & Pied Kingfisher & \multicolumn{4}{|c|}{ Family: Cerylidae } \\
\hline \multicolumn{6}{|l|}{ Family: Coraciidae } \\
\hline \multicolumn{4}{|l|}{ Family: Meropidae } & $\mathrm{Fc}$ & W \\
\hline \multicolumn{6}{|l|}{$\begin{array}{l}\text { Order: Cuculiformes } \\
\text { Family: Centropodidae }\end{array}$} \\
\hline Hierococcyx varius & Common Hawk Cuckoo & $\mathrm{R}$ & V & $\mathrm{Fc}$ & W \\
\hline Cuculus micropterus & Indian Cuckoo & $\mathrm{R}$ & $\mathrm{V}, \mathrm{T}$ & $\mathrm{Fc}$ & $\mathrm{N}, \mathrm{NE}, \mathrm{NW}$ \\
\hline Eudynamys scolopacea & Asian Cuckoo & $\mathrm{R}$ & $\mathrm{T}$ & $\mathrm{C}$ & W \\
\hline Centropus sinensis & Greater Coucal & $\mathrm{R}$ & $\mathrm{T}$ & Fc & W \\
\hline \multicolumn{6}{|l|}{$\begin{array}{l}\text { Order: Psittaciformes } \\
\text { Family: Psittacidae }\end{array}$} \\
\hline Psittacula krameri & Roseringed Parakeet & $\mathrm{R}$ & V & $\mathrm{Fc}$ & W \\
\hline \multicolumn{6}{|l|}{$\begin{array}{l}\text { Order: Apodiformes } \\
\text { Family: Apodidae }\end{array}$} \\
\hline Cypsiurus balasiensis & Asian Palm Swift & $\mathrm{R}$ & ht & Vc & W \\
\hline Apus affinis & House Swift & $\mathrm{R}$ & ht & Vc & W \\
\hline \multicolumn{6}{|l|}{$\begin{array}{l}\text { Order: Strigiformes } \\
\text { Family: Strigidae }\end{array}$} \\
\hline Athene brama & Spotted owlet & $\mathrm{R}$ & J & $\mathrm{Ra}$ & W \\
\hline \multicolumn{6}{|l|}{$\begin{array}{l}\text { Order: Columbiformes } \\
\text { Family: Columbidae }\end{array}$} \\
\hline Columba livia & Rock Pigeon & $\mathrm{R}$ & ht & $\mathrm{C}$ & $\mathrm{Cn}$ \\
\hline Streptopelia tranquebarica & Red Collared Dove & $\mathrm{R}$ & $\mathrm{T}, \mathrm{V}$ & $\mathrm{Ra}$ & $\mathrm{Cn}, \mathrm{N}$ \\
\hline Streptopelia chinensis & Spotted Dove & $\mathrm{R}$ & $\mathrm{T}, \mathrm{V}, \mathrm{J}$ & $\mathrm{C}$ & W \\
\hline Streptopelia decaocto & Eurasian Collared Dove & $\mathrm{R}$ & $\mathrm{T}, \mathrm{V}, \mathrm{J}$ & C & NE,NW \\
\hline \multicolumn{6}{|l|}{$\begin{array}{l}\text { Order: Gruiformes } \\
\text { Family: Rallidae }\end{array}$} \\
\hline Amaurornis phoenicurus & White-breasted Waterhen & $\mathrm{R}$ & $\mathrm{P}, \mathrm{We}$ & $\mathrm{Fc}$ & W \\
\hline \multicolumn{6}{|l|}{$\begin{array}{l}\text { Order: Ciconiiformes } \\
\text { Family: Scolopacidae }\end{array}$} \\
\hline Actitis hypoleucos & Common Sandpiper & $M$ & $\mathrm{~S}$ & C & W \\
\hline Tringa glareola & Wood Sandpiper & $M$ & re & $\mathrm{Ra}$ & NE, NW \\
\hline Tringa stagnatilis & Marsh Sandpiper & M & re & $\mathrm{Ra}$ & $\mathrm{NE}, \mathrm{NW}$ \\
\hline Calidris minuta & Little Stint & $\mathrm{M}$ & $S$ & $\mathrm{Ra}$ & NW \\
\hline Tringa nebularia & Greenshank & M & re & $\mathrm{Ra}$ & NE, NW \\
\hline Tringa totanus & Common Redshank & $\mathrm{M}$ & re & $\mathrm{Ra}$ & NE, NW \\
\hline \multicolumn{6}{|l|}{ Family: Glareolidae } \\
\hline Charadrius alexandrinus & Kentish Plover & M & $\mathrm{S}$ & $\mathrm{Fc}$ & NW \\
\hline Charadrius dubius & Little Ringed Plover & $\mathrm{M}$ & $S$ & Vc & W \\
\hline Charadrius mongolus & Lesser Sand Plover & $M$ & re & $\mathrm{Fc}$ & SE \\
\hline Charadrius placidus & Long-billed Ringed Plover & $\mathrm{M}$ & re, S & $\mathrm{Ra}$ & $\mathrm{Cn}, \mathrm{NE}$ \\
\hline
\end{tabular}




\begin{tabular}{|c|c|c|c|c|c|}
\hline Scientific name & Common Name & $\begin{array}{l}\text { Migratory (M) / } \\
\text { Resident (R) }\end{array}$ & Habitat & Local status & Local Distribution \\
\hline \multicolumn{6}{|l|}{ Family: Accipitridae } \\
\hline Milvus migrans & Black Kite & $\mathrm{R}$ & ht & $\mathrm{C}$ & W \\
\hline Haliastur indus & Brahminy Kite & $\mathrm{R}$ & V & $\mathrm{Ra}$ & NE, NW \\
\hline Accipiter badius & Shikra & $\mathrm{R}$ & $\mathrm{V}, \mathrm{T}$ & $\mathrm{Ra}$ & $\mathrm{N}$ \\
\hline Circus macrourus & Pallid Harrier & $\mathrm{M}$ & $\mathrm{T}$ & $\mathrm{Ra}$ & $\mathrm{Cn}$ \\
\hline $\begin{array}{l}\text { Family: Falconidae } \\
\text { Falco tinnunculus }\end{array}$ & Common Kestrel & $\mathrm{M}$ & $\mathrm{V}, \mathrm{O}$ & $\mathrm{Ra}$ & W \\
\hline $\begin{array}{l}\text { Family: Phalacrocoracic } \\
\text { Phalacrocorax niger } \\
\text { Phalacrocorax carbo }\end{array}$ & $\begin{array}{l}\text { Little Cormorant } \\
\text { Great Cormorant }\end{array}$ & $\begin{array}{l}\mathrm{R} \\
\mathrm{R}\end{array}$ & $\begin{array}{l}r \\
r\end{array}$ & $\begin{array}{l}\mathrm{C} \\
\mathrm{Ra}\end{array}$ & $\begin{array}{l}\text { W } \\
\text { Cn }\end{array}$ \\
\hline $\begin{array}{l}\text { Family: Ardeidae } \\
\text { Egretta garzetta } \\
\text { Mesophoyx intermedia } \\
\text { Casmerodius albus } \\
\text { Bubulcus ibis } \\
\text { Ardea cinerea } \\
\text { Ardeola grayii }\end{array}$ & $\begin{array}{l}\text { Little Egret } \\
\text { Intermediate Egret } \\
\text { Great Egret } \\
\text { Cattle Egret } \\
\text { Grey Heron } \\
\text { Indian Pond Heron }\end{array}$ & $\begin{array}{l}\mathrm{R} \\
\mathrm{R} \\
\mathrm{R} \\
\mathrm{R} \\
\mathrm{R} \\
\mathrm{R}\end{array}$ & $\begin{array}{l}\text { we } \\
\text { we } \\
\text { re } \\
\text { pf, re } \\
\text { re } \\
\mathrm{P}, \mathrm{r}\end{array}$ & $\begin{array}{l}\text { Vc } \\
\mathrm{C} \\
\mathrm{Ra} \\
\mathrm{C} \\
\mathrm{Ra} \\
\mathrm{Vc}\end{array}$ & $\begin{array}{l}\mathrm{W} \\
\mathrm{W} \\
\mathrm{N} \\
\mathrm{W} \\
\mathrm{N} \\
\mathrm{W}\end{array}$ \\
\hline $\begin{array}{l}\text { Order: Passeriformes } \\
\text { Family: Irenidae } \\
\text { Chloropsis aurifrons }\end{array}$ & Golden-fronted Leafbird & $\mathrm{R}$ & $\mathrm{V}, \mathrm{J}$ & $\mathrm{Ra}$ & W \\
\hline $\begin{array}{l}\text { Family: Laniidae } \\
\text { Lanius schach } \\
\text { Lanius cristatus }\end{array}$ & $\begin{array}{l}\text { Long-tailed Shrike } \\
\text { Brown Shrike }\end{array}$ & $\begin{array}{l}\mathrm{R} \\
\mathrm{M}\end{array}$ & $\begin{array}{l}\mathrm{V} \\
\mathrm{V}\end{array}$ & $\begin{array}{l}\mathrm{C} \\
\mathrm{Fc}\end{array}$ & $\begin{array}{l}\text { W } \\
W\end{array}$ \\
\hline $\begin{array}{l}\text { Family: Corvidae } \\
\text { Dendrocitta vagabunda } \\
\text { Corvus splendens } \\
\text { Corvus macrorhynchos } \\
\text { Oriolus chinensis } \\
\text { Oriolus xanthornus } \\
\text { Pericrocotus ethologus } \\
\text { Pericrocotus flammeus } \\
\text { Dicrurus macrocercus } \\
\text { Dicrurus leucophaeus } \\
\text { Aegithina tiphia }\end{array}$ & $\begin{array}{l}\text { Rufous Treepie } \\
\text { House Crow } \\
\text { Jungle Crow } \\
\text { Black-naped Oriole } \\
\text { Black-headed Oriole } \\
\text { Long-tailed Minivet } \\
\text { Scarlet Minivet } \\
\text { Black Drongo } \\
\text { Grey Drongo } \\
\text { Common lora }\end{array}$ & $\begin{array}{l}R \\
R \\
R \\
M \\
R \\
M \\
R \\
R \\
M \\
R\end{array}$ & $\begin{array}{l}\text { J, V } \\
\text { ht } \\
\text { V } \\
\text { V } \\
\text { V, ht } \\
\text { ri } \\
\text { V, ri } \\
\text { ht } \\
\text { T,J } \\
\text { V, J }\end{array}$ & $\begin{array}{l}\mathrm{C} \\
\mathrm{FC} \\
\mathrm{C} \\
\mathrm{Ra} \\
\mathrm{C} \\
\mathrm{C} \\
\mathrm{Ra} \\
\mathrm{Vc} \\
\mathrm{FC} \\
\mathrm{C}\end{array}$ & $\begin{array}{l}\text { Cn, SE, SW } \\
\text { W } \\
\text { W } \\
\text { SE } \\
\text { W } \\
\text { NE, NW } \\
\text { Cn, NE } \\
\text { W } \\
\text { W } \\
\text { W }\end{array}$ \\
\hline $\begin{array}{l}\text { Family: Muscicapidae } \\
\text { Turdus naumanni } \\
\text { Zoothera citrina } \\
\text { Muscicapa dauurica } \\
\text { Culicicapa ceylonensis } \\
\text { Luscinia svecica } \\
\text { Copsychus saularis } \\
\text { Phoenicurus ochruros } \\
\text { Heterophasia picaoides }\end{array}$ & $\begin{array}{l}\text { Dusky Thrush } \\
\text { Orange-headed Thrush } \\
\text { Asian Brown Flycatcher } \\
\text { Grey-headed canary Flycatcher } \\
\text { Bluethroat } \\
\text { Oriental Magpie Robin } \\
\text { Black Redstart } \\
\text { Long-tailed Sibia }\end{array}$ & $\begin{array}{l}M \\
M \\
M \\
R \\
M \\
R \\
M \\
M\end{array}$ & $\begin{array}{l}\text { J } \\
\text { V } \\
\text { V, ri } \\
\text { V } \\
\text { ri } \\
\text { ht } \\
\text { ri } \\
\text { V, J }\end{array}$ & $\begin{array}{l}\mathrm{Ra} \\
\mathrm{Ra} \\
\mathrm{Fc} \\
\mathrm{Fc} \\
\mathrm{Ra} \\
\mathrm{C} \\
\mathrm{Ra} \\
\mathrm{FC}\end{array}$ & $\begin{array}{l}\text { NW } \\
\text { Cn, NW } \\
\text { NE, NW } \\
\text { W } \\
\text { SE, SW } \\
\text { W } \\
\text { NE } \\
\text { NE }\end{array}$ \\
\hline $\begin{array}{l}\text { Family: Sturnidae } \\
\text { Sturnus contra } \\
\text { Sturnus malabaricus } \\
\text { Acridotheres tristis } \\
\text { Acridotheres fuscus } \\
\text { Acridotheres ginginianus }\end{array}$ & $\begin{array}{l}\text { Asian Pied Starling } \\
\text { Chestnut-tailed Starling } \\
\text { Common Myna } \\
\text { Jungle Myna } \\
\text { Bank Myna }\end{array}$ & $\begin{array}{l}R \\
R \\
R \\
R \\
R\end{array}$ & $\begin{array}{l}\text { ht } \\
\text { V } \\
\text { ht } \\
\text { V, J } \\
\text { L }\end{array}$ & $\begin{array}{l}\text { Vc } \\
\text { Fc } \\
\mathrm{C} \\
\mathrm{C} \\
\mathrm{Ra}\end{array}$ & $\begin{array}{l}\text { W } \\
\text { SW } \\
\text { W } \\
\text { W } \\
\text { NW }\end{array}$ \\
\hline $\begin{array}{l}\text { Family: Paridae } \\
\text { Parus major } \\
\text { Riparia riparia }\end{array}$ & $\begin{array}{l}\text { Great Tit } \\
\text { Sand Martin }\end{array}$ & $\begin{array}{l}\mathrm{R} \\
\mathrm{M}\end{array}$ & $\begin{array}{l}\mathrm{V} \\
\mathrm{V}\end{array}$ & $\begin{array}{l}\text { Fc } \\
\text { Ra }\end{array}$ & $\begin{array}{l}\text { W } \\
W\end{array}$ \\
\hline $\begin{array}{l}\text { Family: Hirundinidae } \\
\text { Hirundo rustica }\end{array}$ & Barn Swallow & $\mathrm{M}$ & V & Vc & W \\
\hline $\begin{array}{l}\text { Family: Pycnonotidae } \\
\text { Pycnonotus cafer } \\
\text { Pycnonotus jocosus }\end{array}$ & $\begin{array}{l}\text { Red-vented Bulbul } \\
\text { Red-whiskered Bulbul }\end{array}$ & $\begin{array}{l}\mathrm{R} \\
\mathrm{R}\end{array}$ & $\begin{array}{l}\text { V, ht } \\
\text { ht }\end{array}$ & $\begin{array}{l}\text { Vc } \\
\text { Fc }\end{array}$ & $\begin{array}{l}\text { W } \\
\text { Cn, SE }\end{array}$ \\
\hline $\begin{array}{l}\text { Family: Cisticolidae } \\
\text { Prinia inornata } \\
\text { Prinia sylvatica } \\
\text { Prinia flaviventris } \\
\text { Prinia gracilis }\end{array}$ & $\begin{array}{l}\text { Plain Prinia } \\
\text { Jungle Prinia } \\
\text { Yellow-bellied Prinia } \\
\text { Graceful Prinia }\end{array}$ & $\begin{array}{l}\mathrm{R} \\
\mathrm{R} \\
\mathrm{R} \\
\mathrm{R}\end{array}$ & $\begin{array}{l}\mathrm{ri} \\
\mathrm{ri} \\
\mathrm{ri} \\
\mathrm{ri}\end{array}$ & $\begin{array}{l}\mathrm{C} \\
\mathrm{Vc} \\
\mathrm{Ra} \\
\mathrm{Vc}\end{array}$ & $\begin{array}{l}\text { NE, NW } \\
\text { NE, NW } \\
\text { NE } \\
\text { NE, NW }\end{array}$ \\
\hline $\begin{array}{l}\text { Family: Zosteropidae } \\
\text { Zosterops palpebrosus }\end{array}$ & Oriental White-eye & $\mathrm{R}$ & V & $\mathrm{C}$ & W \\
\hline $\begin{array}{l}\text { Family: Sylviidae } \\
\text { Phylloscopus fuscatus } \\
\text { Orthotomus sutorius } \\
\text { Malacocincla abbotti } \\
\text { Turdoides striatus }\end{array}$ & $\begin{array}{l}\text { Dusky Warbler } \\
\text { Common Tailorbird } \\
\text { Abbot's Babbler } \\
\text { Jungle Babbler }\end{array}$ & $\begin{array}{l}M \\
R \\
R \\
R\end{array}$ & $\begin{array}{l}\mathrm{ri} \\
\mathrm{V} \\
\mathrm{V}, \mathrm{J}\end{array}$ & $\begin{array}{l}\text { Fc } \\
\mathrm{C} \\
\mathrm{Ra} \\
\mathrm{C}\end{array}$ & $\begin{array}{l}\text { NE, NW } \\
\text { W } \\
\text { SW } \\
\text { W }\end{array}$ \\
\hline
\end{tabular}




\begin{tabular}{|c|c|c|c|c|c|}
\hline Scientific name & Common Name & $\begin{array}{l}\text { Migratory (M) / } \\
\text { Resident (R) }\end{array}$ & Habitat & Local status & Local Distribution \\
\hline \multicolumn{6}{|l|}{ Family: Alaudidae } \\
\hline Mirafra erythroptera & Indian Bushlark & $\mathrm{R}$ & $J$ & $\mathrm{Fc}$ & W \\
\hline Calandrella raytal & Sand Lark & $\mathrm{R}$ & $\mathrm{S}$, re & $\mathrm{Ra}$ & NW \\
\hline \multicolumn{6}{|l|}{ Family: Nectariniiae } \\
\hline Dicaeum erythrorynchos & Pale-billed Flowerpecker & $\mathrm{R}$ & $\mathrm{V}$ & C & W \\
\hline Dicaeum concolor & Plain Flowerpecker & $\mathrm{R}$ & v & $\mathrm{Fc}$ & SE \\
\hline Nectarinia asiatica & Purple Sunbird & $\mathrm{R}$ & $\mathrm{V}, \mathrm{J}$ & $\mathrm{C}$ & W \\
\hline \multicolumn{6}{|l|}{ Family: Fringillidae } \\
\hline Emberiza pusilla & Liitle Bunting & M & V & $\mathrm{Ra}$ & NW, SW \\
\hline \multicolumn{6}{|l|}{ Family: Passeridae } \\
\hline Passer domesticus & House Sparrow & $\mathrm{R}$ & $\mathrm{ht}$ & $V_{c}$ & W \\
\hline Motacilla flava & Yellow Wagtail & M & $\mathrm{S}, \mathrm{re}$ & $\mathrm{Ra}$ & NE, NW \\
\hline Motacilla alba & White Wagtail & M & $\mathrm{s}, \mathrm{re}$ & Vc & W \\
\hline Motacilla cinerea & Grey Wagtail & M & S, re & $\mathrm{Fc}$ & W \\
\hline Anthus rufulus & Paddyfield Pipit & M & ri & $\mathrm{Ra}$ & NW \\
\hline Ploceus philippinus & Baya weaver & $\mathrm{R}$ & $\mathrm{V}$ & $\mathrm{C}$ & W \\
\hline Lonchura malacca & Black-headed Munia & $\mathrm{R}$ & $\mathrm{V}$ & $\mathrm{Fc}$ & sw \\
\hline Lonchura punctulata & Scaly-breasted Munia & $\mathrm{R}$ & V, ri & $\mathrm{Ra}$ & sw \\
\hline
\end{tabular}

Habitat: $\mathrm{r}$ - River; re - River edge; T - Tree; J - Jungle; $\mathrm{V}$ - Village area; We - Water edge; $\mathrm{P}$ - Pond, S - Sand; pf - Paddy field; L - Land; O - Open field; ri - River island; ht - Homestead area

Local status: Ra - Rare; Fc - Fairly common; Vc - Very common; C - Common

Local Distribution: W - Wide; N - North; NE - Northeast; NW - Northwest; SE - Southeast; SW - Southwest; Cn - Central

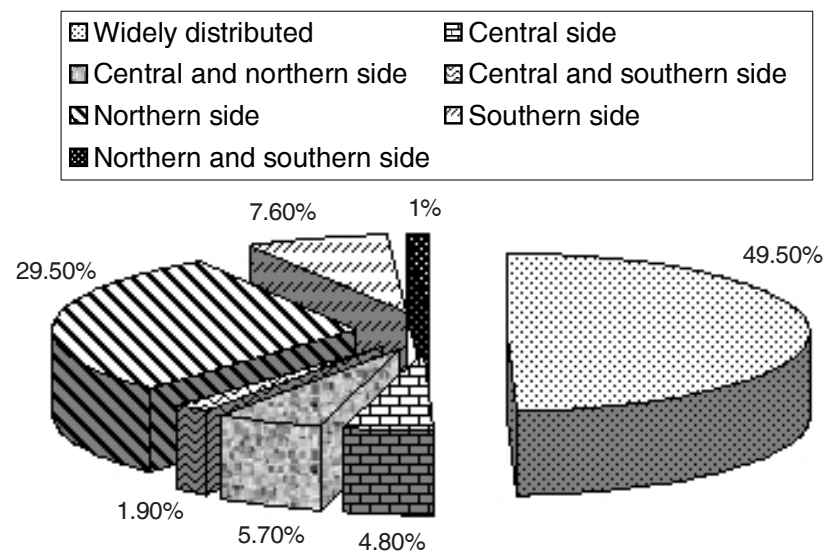

Figure 2. Distribution of birds in the study area

riverside areas, ponds, water edges, beaches etc. The rest, 75 species (71.4\%), were observed on land, in agricultural fields, trees, village, bush, etc. Many aquatic and semi-aquatic species used terrestrial habitats and vice versa. Terrestrial areas supported more species because the areas were favorable for feeding and nesting purposes and had comparatively good vegetation.

Species distribution was not the same throughout the study area. Out of 105 species, $52(49.5 \%)$ were widely distributed in Kurigram District (Fig. 2). From the remaining 53 (50.5\%) species, $31(29.5 \%)$ were restricted only to the northern side, fiveight $(4.8 \%)$ to the central side, and $8(7.6 \%)$ to the southern side. Nine $(8.6 \%)$ species were common in two or three parts of the study area among which six $(5.7 \%)$ were recorded from the central and northern sides, two (1.9\%) were from the central and southern sides, and only one (1\%) was common in the northern and southern parts of the study area. From our observations it was apparent that the northern side of the study area supported more species because it was far away from human habitation and less disturbed.

Among the three canopy levels, 16 (15.2\%) species were recorded in lower canopy, $32(30.5 \%)$ were recorded in both lower and middle canopies, $19(18.1 \%)$ species in upper and middle canopies, and only $1(1 \%)$ species in upper canopy. Thirty-seven (35.2\%) species used all three canopy levels.

A total of 44 species of plants were observed being used by birds. Among these $15(34.1 \%)$ were fruiting trees and 18 $(40.9 \%)$ woody trees, $10(22.7 \%)$ were shrubs, and only $1(2.3 \%)$ was an aquatic species. Out of 105 species, 30 (28.6\%) were predominantly ground dwelling birds, five (4.8\%) species were observed on fruiting trees, four $(3.8 \%)$ species were observed on woody trees, and 16 (15.2\%) species were observed on shrubs only. Five $(4.8 \%)$ species of birds used all types of plants species. Each of the cultivated fields and aquatic vegetation contained only a single (1\%) species.

From direct observation it was found that most of the birds eat a variety of food types. When a bird eats a single item of food more than 60 percent of the time, then we considered it as the main food type for that species. Of the total (105) species of birds, $48(45.7 \%)$ were insectivorous, $11(10.4 \%)$ grainivorous, five $(4.8 \%)$ frugivorous, $10(9.5 \%)$ piscivorous, five $(4.8 \%)$ predatory, and $19(18.1 \%)$ species of birds were omnivorous. Only one (1\%) was vegetarian and the foods of six $(5.7 \%)$ species could not be determined.

\section{References}

Ali, S. (1996). The Book of Indian Birds (12 $2^{\text {th }}$ revised and centenary edition). Bombay Natural History Society, New Delhi, $345 \mathrm{pp}$.

Ali, S. \& S. D. Ripley (1983). A Pictorial Guides to the Birds of the Indian Subcontinent. Bombay Natural History society, New Delhi. ii $+177 \mathrm{pp}$.

Grimmett, R., C. Inskipp \& T. Inskipp (1999). Pocket Guide to the Birds of the Indian Subcontinent. Oxford University Press. New Delhi, 888pp.

Harvey, W.G. (1990). Birds in Bangladesh. University Press Limited. Dhaka. viii +188 pp.

Inskipp, T., N. Lindsey \& W. Duckworth (1996). An Annotated Checklist of the Birds of the Oriental Region. Oriental Bird Club. Berdfordshire, 294pp.

IUCN (2000). Red Book of Threatened Birds of Bangladesh. IUCN-The World Conservation Union, xii $+116 \mathrm{pp}$.

Khan, M.A.R. (1982). Wildlife of Bangladesh: A Checklist. University of 
Dhaka, Dhaka, iv + 174pp.

Khan, M.A.R. (1980). A Comparative account of the avifauna of the shoals and the neighbouring plantation in the Nilgiri. Journal of the Bombay Natural History Society 75(3): 1028-1035.

Khan, M.M.H. (2005). Species diversity, relative abundance and habitat use of the birds in the Sundarbans East Wildlife Sanctuary,
Bangladesh. Forktail (21): 79-86.

Thompson, P.M. \& D.L. Johnson (2003). Further notable bird records from Bangladesh. Forktail (19): 85-102. 